\title{
Primary soft tissue angiomyolipoma in a dog with unusual clinical features
}

\author{
Jiř́i Lenz ${ }^{1,2,3}$, Petra Konečná1, František Tichý ${ }^{1}$, Luděk Fiala ${ }^{3,4}$ \\ ${ }^{1}$ University of Veterinary Sciences Brno, Faculty of Veterinary Medicine, Department of Anatomy, \\ Histology and Embryology, Brno, Czech Republic \\ ${ }^{2}$ Znojmo Hospital, Department of Pathology, Znojmo, Czech Republic \\ ${ }^{3}$ Cytohisto s.r.o., Břeclav, Czech Republic \\ ${ }^{4}$ Charles University Prague, First Faculty od Medicine, Prague, Czech Republic \\ Received October 24, 2020 \\ Accepted May 26, 2021
}

\begin{abstract}
Angiomyolipoma is an extremely rare neoplasm in animals. It belongs to a group of perivascular epithelioid cell tumours (so-called PEComas). This study reports a case of primary soft tissue angiomyolipoma in a dog with some unusual clinical features. A 4-year old female Labrador dog with a rapidly growing pelvic tumour measuring $30 \times 20 \mathrm{~cm}$ with a short history of 12 weeks was presented. The tumour was well-circumscribed and pushed into the right vaginal wall and into the perineum. The tumour was completely surgically excised. An extensive histological examination was performed, including immunohistochemical analysis. Histology revealed a mesenchymal neoplasm consisting of three tissue components - mature adipose tissue (which dominated), vessels, and smooth muscle (spindle) cells. The lesion showed positive immunohistochemical staining with smooth muscle actin and desmin in mature-appearing smooth muscle cells and S-100 protein positivity in adipocytes.

The diagnosis of angiomyolipoma must be considered in different types of benign and malignant tumours of various lineages. The diagnostic approach to soft tissue tumours, including angiomyolipoma, requires optimal processing and sectioning of resected specimens. This paper is believed to be the first case of primary soft tissue angiomyolipoma reported in the veterinary literature.
\end{abstract}

Perivascular epithelioid cell tumour, animal, immunohistochemistry

Angiomyolipoma (AML) belongs to a group of perivascular epithelioid cell tumours referred to as PEComas (tumours showing perivascular epithelioid cell tumours). The concept of this tumour family was developed by Bonetti et al. (1992). The authors found that some neoplasms located at different sites are composed of cells that appear to originate from the wall of the vessels and exhibit similar histopathological characteristics. The defining feature is the presence of cells showing both myoid and melanocytic differentiation demonstrated by immunohistochemistry.

Microscopically, classic AML is a triphasic tumour composed of a mixture of mature adipose tissue, vessels, and smooth muscle (spindle) cells. Adipocytes are often the predominant cell type, lacking atypia, but their size can vary widely. Multivacuolated cells suggesting lipoblasts may also be present (Hruban et al. 1989). The vascular component consists of abnormal (poorly organized) blood vessels with an eccentrically thickened wall, often surrounded by periadventitial clusters of spindle and/or epithelioid cells (Folpe et al. 2005). In a minority of cases, the smooth muscle (PEComa) component predominates. Its histological appearance ranges from mature smooth muscle cells, which exhibit eosinophilic cytoplasm and uniform blunt-ended, cigar-shaped nuclei, to immature spindle cells, arranged in a radial fashion around the vessels, in solid sheets or fascicles (Nonomura et al. 1998).

In dogs, the only known site of involvement by AML is the uterine horn (Boisclair and Doré 2001).

Address for correspondence:

Jiři Lenz

Department of Pathology, Znojmo Hospital

MUDr. Jana Janského 11

Znojmo, 669 02, Czech Republic

E-mail: jiri.lenz@gmail.com

Phone: +420515215478

http://actavet.vfu.cz/ 
Due to the rarity of AML (PEComas), no minimal diagnostic criteria for malignancy have been established. However, features such as tumour necrosis, large size, mitotic activity, marked nuclear atypia and pleomorphism should raise the suspicion of malignancy (Folpe et al. 2005; Folpe and Kwiatkowski 2010).

Herein, a case of primary soft tissue angiomyolipoma of the pelvis in a dog with some unusual clinical features is presented.

\section{Case description}

A 4-year-old intact female dog with a rapidly growing pelvic mass was presented. All clinical examinations, including ultrasound and surgery, were performed at the Department of Surgery and Orthopaedics of the Faculty of Veterinary Medicine, University of Veterinary Sciences Brno. First, a clinical examination was performed with the following results. A painless mass of soft consistency spherically displacing the perineum was present. The vagina and anus were slightly swollen and congested, and no signs of vaginal or uterine prolapse were detected. The bitch was an otherwise healthy Labrador with a short history of tumour lasting 12 weeks (no pain, bleeding or other symptoms were observed). In the next step, on the same day, an ultrasound examination was indicated. An abdominal and pelvic ultrasound revealed a large $30 \times 20 \mathrm{~cm}$ tumour located on the right lateral wall of the vagina extending into the perineum. The lesion was richly-vascularized, relatively well-circumscribed and seemed to originate from the muscle layer of the vaginal wall and cervix uteri. No other imaging method was indicated. All laboratory test results were within normal ranges. Subsequently, the following day, surgery was performed and the tumour was completely removed. One day after the operation, the patient was discharged in a stable condition. The tumour was extensively examined both macroscopically and microscopically. Grossly, the surgically excised specimen consisted of well-demarcated, uncapsulated mass with a pale yellow cut surface. Necrosis, cystic changes or haemorrhage were not present. The specimen was extensively investigated using 30 tissue sections stained with haematoxylin-eosin. Histological examination revealed mesenchymal neoplasm composed of an admixture of adipose tissue, vessels and smooth muscle (spindle) cells. The adipose tissue was mature and formed a major proportion of the lesion. Minor areas consisting of bundles of smooth muscle cells, with focally immature appearance, and thickwalled, medium to large sized vessels were focally present (Plate VII, Fig. 1A-D). No pleomorphism, nuclear atypia, increased mitotic activity, arborating capillary vasculature, myxoid regions or metaplastic changes were seen. The resected specimen of our case did not contain parts of the vaginal wall or any other pelvic organ.

To determine the nature of the lesion, special staining methods were performed. Immunohistochemical staining was performed on the automated immunostainer BenchMark Ultra (Ventana Medical System Inc, Oro Valley, USA) on formalin-fixed $(10 \%$ neutral buffered formalin; fixation time $24 \mathrm{~h})$, paraffin-embedded tissue sections (3 $\mu \mathrm{m}$ thick). The following antibodies were employed: S100 protein (polyclonal, ready to use [RTU], Roche, Basel, Switzerland), HMB-45 (monoclonal, clone HMB-45, RTU, Roche), Melan-A (monoclonal, clone A103, RTU, Roche), smooth muscle actin (SMA) (monoclonal, clone 1a4, RTU, Roche) and desmin (monoclonal, clone DE-R-11, RTU, Roche). The above were visualized using the ultraView Universal DAB Detection Kit (Ventana Medical System Inc). External positive controls were used for all antibodies (human malignant melanoma for S-100 and HMB-45, human leiomyoma for SMA and desmin). Negative controls were prepared by incubating samples without a primary antibody.

Imunohistochemistry revealed coexpression of smooth muscle actin and desmin in mature-appearing smooth muscle cells (Plate VII, Fig. 1E-F). Reactivity for S-100 protein 


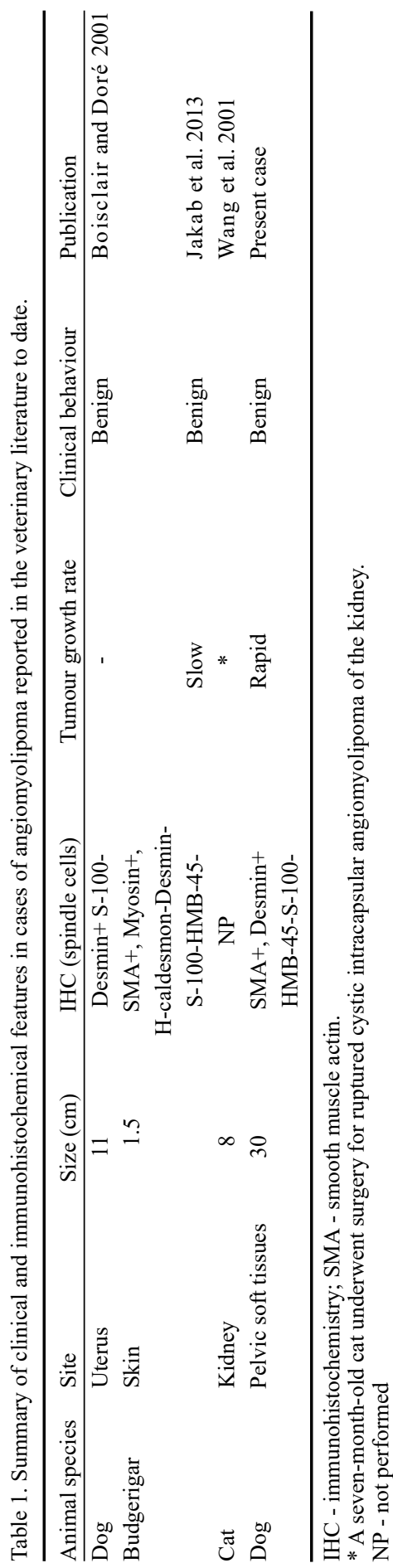

was observed only in adipocytes. Staining with melanocytic markers HMB-45 and Melan-A was completely negative. However, reactivity with the HMB-45 and Melan-A monoclonal antibodies used in our immunohistochemical analysis has not been tested on canine tissue and therefore their negativity cannot be considered valid.

Based on all the previously described morphological and immunohistochemical features observed in this case, a final diagnosis of primary soft tissue angiomyolipoma of the pelvis was made. Clinical behaviour was benign with no signs of recurrence or metastasis at a 4-year follow-up.

\section{Discussion}

Angiomyolipoma is extremely rare in animals. To our knowledge, only 3 cases in different animal species have been published in the veterinary literature so far (Table 1) (Boisclair and Doré 2001; Wang et al. 2001; Jakab et al. 2013). In the study by Boisclair and Doré (2001), it was a large multinodular mass affecting the uterine horn in a dog with a triphasic histology corresponding to AML. Interestingly, multiple foci of metaplastic cartilage and bone were detected. Immunohistochemistry revealed desmin reactivity in smooth muscle cells and S-100 protein reactivity in adipocytes and chondrocytes. Staining with antibody against HMB-45 was not performed. Microscopically, a direct continuity between AML and myometrium was apparent, and therefore, the tumour was classified as primarily uterine. In the remaining 2 cases, the kidney in a cat (Wang et al. 2001) and the skin of a budgerigar (Jakab et al. 2013) were affected. In our case, it was a pelvic tumour that pushed into the right vaginal wall and into the perineum. The lack of an infiltrative growth pattern allowed complete tumour removal. Parts of the vaginal wall or any other pelvic organ were not present in the resected specimen, which was a key finding confirming the primary soft tissue origin.

Regarding the biological potential of the present case, some worrisome clinical features suggesting malignancy were observed, namely, the large tumour size and rapid growth with a short preoperative duration of 12 weeks. The latter represents a very atypical manifestation of AML that has not been described previously. Boisclair and Doré (2001) reported an $11 \mathrm{~cm}$ large uterine AML in a dog; unfortunately, the study lacks data 
concerning the duration of clinical symptoms. In the study by Wang et al. (2001), ruptured cystic intracapsular AML of the kidney in a cat was presented. The remaining case was a slow-growing subcutaneous tumour of a diameter of $1.5 \mathrm{~cm}$ in a budgerigar (Jakab et al. 2013). Searching the available follow-up data, all AMLs, including our case, behaved in a benign manner (metastatic disease or recurrence were not reported).

An appropriate approach is needed when processing soft tissue samples. The resected specimen of our case was completely dissected at regular $1 \mathrm{~cm}$ intervals and then carefully inspected. The tumour tissue was homogeneously pale yellow on sectioning; areas that appeared grossly distinct were not detected. We were aware that the tumour could have heterogeneous appearance on histological examination and therefore, a large volume of the resected tissue was submitted (a total of 30 sections). This approach was key to making the correct diagnosis. The tumour was originally diagnosed by another pathologist as a benign lipoma. Because the gross appearance did not suggest malignancy, only one tissue block was submitted in which mature adipose tissue was found. As shown in this example, tumours with any suspicious features (morphological as well as clinical) should be carefully processed and submitted to ensure accurate diagnosis.

The morphological appearance of AML is usually characteristic and does not cause problems in histological diagnosis. However, in certain clinical scenarios, AML may be misdiagnosed as sarcoma or carcinoma. The differential diagnostic spectrum of AML is variable depending on the proportion of its individual components. Findings such as variation in adipocyte size and scattered lipoblasts may be confusing in tumours with a predominant fatty component. If the PEComa component is not readily apparent, these tumours can easily be mistaken for a well-differentiated liposarcoma (a lipoma-like subtype) (Hruban et al. 1989). The distinction is based on the lack of adipocytic nuclear atypia and the positivity of melanocytic markers in smooth muscle cells in AML. Therefore, thorough sampling is required to confirm the presence of the smooth muscle (spindle) cell component in AML. Another challenging differential diagnosis is encountered in cases of spindled mesenchymal-appearing tumours. Leiomyosarcoma may be difficult to distinguish from AML with an inconspicuous fatty component; features favouring leiomyosarcoma are high cellularity, frequent mitotic activity, and significant cytologic atypia. In renal AML, the epithelioid variant should be differentiated from renal cell carcinoma. Both tumours have an epithelioid morphology and consist of cells with abundant granular eosinophilic and/or clear cytoplasm and round nuclei. Despite cytological similarities, intratumoral fat is exceedingly rare in renal cell carcinoma. Immunohistochemical examination combining melanocytic markers with keratins and EMA is of high utility in resolving the differential diagnosis between epithelioid angiomyolipoma and renal cell carcinoma.

Positivity for melanocytic markers can often point to metastatic melanoma. Clinical information, such as history of melanoma or multinodular growth, can provide a clue to the correct diagnosis. Both entities can be distinguished with certainty based on smooth muscle actin expression (positive in AML and negative in melanoma).

The list of lesions that may be considered in the differential diagnosis of AML also includes benign processes such as lipoma, angiolipoma, angioleiomyoma, myolipoma and myelolipoma. Lipoma consists exclusively of mature adipocytes, while in angiolipoma, capillary-sized vessels are seen in a background of an adipose tissue. Angioleiomyoma is characterized by mature smooth muscle cells with intervening vascular spaces and a lack of adipose tissue. In myolipoma, only smooth muscle cells and adipocytes are found. Myelolipoma consists of two tissue components - bone marrow elements and mature adipose tissue.

In addition to AML, the group of PEComas include lymphangioleiomyomatosis, clear cell "sugar" tumour of the lung and a number of tumours sharing similar morphological and immunohistochemical features arising in a wide variety of soft-tissue and visceral sites 
(Folpe and Kwiatkowski 2010). Some human PEComas are associated with tuberous sclerosis complex (Eble 1998).

In terms of the biological nature, AML was originally considered a hamartomatous proliferation. However, recent molecular data established AML as a clonal neoplasm. Genetic studies have revealed that changes in the tuberous sclerosis complex (TSC) play a key role in the oncogenesis of PEComas (Green et al. 1994). Tuberous sclerosis complex is a genetic disease caused by mutations in the TSC 1 or TSC 2 genes, which encodes hamartin and tuberin, proteins involved in certain cellular activities including cell proliferation and differentiation. Loss of these genes leads to activation of the mTOR/p70S6K signalling pathway (Kenerson et al. 2007). In both sporadic AML and those associated with TSC, there is usually a loss of heterozygosity of the TSC2 gene, while allelic losses of the TSC1 gene occur rather occasionally (Pan et al. 2008).

In conclusion, the correct classification of AML is difficult due to its rarity, variable morphological findings and some clinical features (large size, rapid growth). The diagnosis of AML must be considered in different types of benign and malignant tumours of various lineages (mesenchymal, especially lipomatous and spindle cell, as well as epithelial). The diagnostic approach to soft tissue tumours requires careful evaluation of macroscopic findings and optimal processing and sectioning of resected samples.

To the athors' knowledge, this study is the first case of primary soft tissue angiomyolipoma reported in the veterinary literature.

\section{References}

Boisclair J, Doré M 2001: Uterine angiolipoleiomyoma in a dog. Vet Pathol 38: 726-728

Bonetti F, Pea M, Martignoni G, Zamboni G 1992: PEC and sugar. Am J Surg Pathol 16: 307-308

Eble JN 1998. Angiomyolipoma of the kidney. Semin Diagn Pathol 15: 21-40

Folpe AL, Mentzel T, Lehr HA, Fischer C, Balzer BL, Weiss SW 2005: Perivascular epithelioid cell neoplasms of soft tissue and gynecologic origin: a clinicopathologic study of 26 cases and review of the literature. Am J Surg Pathol 29: 1558-1575

Folpe AL, Kwiatkowski DJ 2010. Perivascular epithelioid cell neoplasms: pathology and pathogenesis. Hum Pathol 41: 1-15

Green AJ, Smith M, Yater RRW 1994: Loss of heterozygosity on chromosome 16p13.3 in hamartomas from tuberous sclerosis patients. Nature Genet 6: 193-196

Hruban RH, Bhagavan BS, Epstein JH 1989: Massive retroperitoneal angiomyolipoma: A lesion that may be confused with well-differentiated liposarcoma. Am J Clin Pathol 92: 805-808

Jakab C, Balka G, Szabára A, Csaba C, Pazár P 2013: A case of cutaneous angiolipoleiomyoma (angiomyolipoma) in a budgerigar (Melopsittacus undulatus). Avian Pathology 42: 511-515

Kenerson H, Folpe AL, Takayama TK, Yeung RS 2007: Activation of the mTOR pathway in sporadic angiomyolipomas and other perivascular epithelioid cell neoplasms. Hum Pathol 38: 1361-1371

Nonomura A, Minato H, Kurumaya H 1998: Angiomyolipoma predominantly composed of smooth muscle cells: problems in histological diagnosis. Histopathology 33: 20-27

Pan CC, Chung MY, Ng KF, Liu CY, Wang JS, Chai CY, Huang SH, Chen PCH, Ho DMT 2008: Constant allelic alteration on chromosome 16p (TSC2 gene) in perivascular epithelioid cell tumour (PEComa): genetic evidence for the relationship of PEComa with angiomyolipoma. J Pathol 214: 387-393

Wang FI, Liang SL, Chen GH, Chen DU 2001: Unilateral concurrence of pyelocaliceal diverticula and intracapsular angiomyolipoma in the kidney of a cat. J Vet Diagn Incest 13: 167-169 
Plate VII

Lenz J. et al.: Primary... pp. 179-183

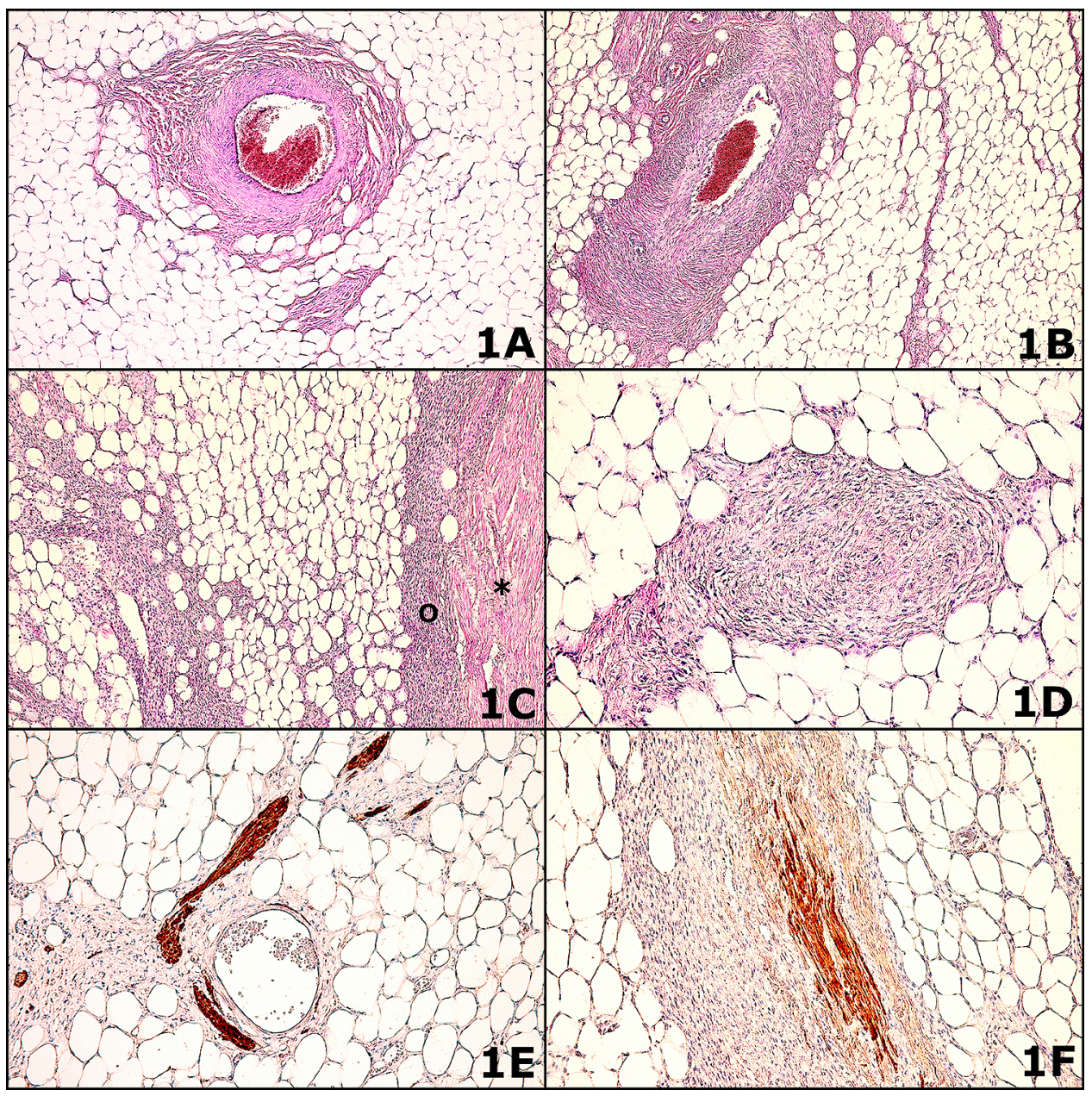

Fig. 1. Morphological and immunohistochemical features of angiomyolipoma

Angiomyolipoma consisting of mature adiposse tissue and thick-walled, medium (A) to large sized vessels (B) with an eccentricaly thickened wall surrounded by periadventitial clusters of spindle cells (haematoxylin-eosin staining, original magnification $\times 100)$. The smooth muscle $($ PEComa) component wih an apparent interface between mature- $(*)$ and immature-appearing $\left({ }^{\circ}\right)$ spindle cells (C) (haematoxylin-eosin staining, original magnification $\times 100$ ). Solid nest of immature-appearing spindle cells (D) (haematoxylin-eosin staining, original magnification $\times 200$ ). Expression of smooth muscle actin $(E)$ and desmin in mature-appearing smooth muscle cells $(\mathrm{F})$ (immunohistochemistry, original magnification $\times 200$ ). 